\title{
Idealization of CAD model for a simulation by a finite element method
}

\author{
Mounir Hamdi - Nizar Aifaoui - Borhen Louhichi \\ Abdelmajid BenAmara \\ Laboratoire de Génie Mécanique \\ Ecole Nationale d'Ingénieurs de Monastir \\ 5 avenue Ibn El Jazzar 5019 Monastir Tunisie \\ nizar.aifaoui@ipeim.rnu.tn
}

\begin{abstract}
Nowadays, numeric simulation becomes one of the most important activities of the product development cycle. To accelerate the design and simulation tasks, it is necessary to adapt the CAD model before the simulation process. This paper presents a method based on an original algorithm in order to adapt and simplify the design geometric model to a simulation by finite elements method. It consists in the idealization of the CAD geometry by eliminating details (holes, chamfers, etc.). These details increase the computing time due to a refined mesh in these details, which are considered as stress hubs, without providing more precision in the simulation. An implementation of the proposed algorithm using the Open Cascade platform is also presented. The last part of this paper presents two examples of mechanical parts, which are simulated before and after idealization. The results of simulation illustrate the major contribution of the proposed method in terms of computing time gain, without so much changing the exactitude of results.

RÉSUMÉ. De nos jours, la simulation numérique est devenue une activité clé dans le cycle de développement des produits. Afin d'accélérer le processus de conception-simulation, il est nécessaire d'adapter le modèle CAO avant d'entamer la phase de simulation. Cet article présente un algorithme original permettant d'adapter et simplifier le modèle géométrique pour la simulation par la méthode des éléments finis. La méthode proposée consiste à idéaliser la géométrie CAO par l'élimination de détails (trou, chanfrein, etc.). Ces détails augmentent le temps de calcul dî au raffinement $d u$ maillage dans ces zones de concentration de contraintes sans apporter plus de précision sur les résultats de calcul. La mise en auvre informatique de l'algorithme a été menée sous la plateforme Open Cascade. La dernière partie de cet article présente des exemples de pièces mécaniques simulés avant et après idéalisation. Les résultats de la simulation illustrent l'apport majeur de la proposition en termes de gain en temps de calcul sans influencer la précision des résultats de calcul.

KEYWORDS: CAD geometry, integration, idealization, simulation, optimization, FEM.

MOTS-CLÉS : géométrie CAO, intégration, idéalisation, simulation, optimisation, $M E F$.
\end{abstract}

DOI:10.3166/EJCM.19.419-439 (C) 2010 Lavoisier, Paris 


\section{Introduction}

Design and mechanical analysis were considered, for a long time, as two independent activities. The designer and the analyst work, each one in his domain, using distinct models. Designers propose the product starting by delivery requirements and using specific designer tools. While the analysts, evaluate the proposals of designers basing on analyses tools. The use of distinct tools obliges the analyst to do again the CAD's geometry in order to be able to evaluate their performances. Any modification made by the designer to CAD model leads the analyst to restart the whole tedious work of geometry's preparation for simulation. There is no way to update the model being used for the analysis starting from $\mathrm{CAD}$ model provided by designers. The analysis time becomes excessively long. The consequences of a modification, in an advanced stage of the design, become disastrous for the analysis. The multiple backward and forward steps between design and analysis increase the validation time of the product and thus its cost. This logic of cohabitation between the two activities, design and analysis (simulation), was quickly suspected. Nowadays, methodology of work and software is in continuous development. Simulation is considered to be the main step of the mechanical product elaboration. It is on the basis of simulation's results that the decisions of validation, re-examination or improvement (optimization) of the design proposals will be considered. Mechanical simulation phase requires a stage of the design model's preparation called a pre-processing phase, and requires also a stage of simulation results' exploitation (tables of values, histograms, Iso-values of stress or deformations, etc.), which is called a post-processing phase. The preprocessing phase is considered as the most important one. Its objective consists in adapting the geometric model (CAD model) by cleaning, gathering subsets, creating a grid, specifying boundary conditions, etc. This phase, according to Lockheed Martin Corporation which is specialised in structural analysis, can consume up to $95 \%$ of the total time of the mechanical analysis (Westphal, 2005). Thus, it is clear that the economy of time and cost by the improvement of the pre-processing phase is a reality (Benamara, 1998).

Our research tasks aim at improving CAD models preparation's phase even before starting the stage of mesh (Aifaoui, 2003). The preparation of a CAD model involves a new step called idealization of the geometry by eliminating details (holes, chamfer, fillet, etc), considered superfluous for simulation (Hamdi et al., 2007). Then these details are zones where the mesh will be automatically refined as they will generate a very important computing time without bringing more precision in the results of simulation (Benhafida et al., 2005). The temporary elimination of these details, since the design phase, in preparation for the simulation, allows important time savings without damaging the results of simulation.

The objective of this paper is the proposition of an original algorithm, which can guide the designer in the phase of the CAD model's preparation for a simulation by the finite elements method. According to the approach suggested, the elimination of the details is based on a representation by Iso-zones enabling the designer to visualize the 
details, candidates for elimination depending on the degree of the detail to eliminate. The process of elimination of the details can be automatic or interactive with the designer. The first part of the article presents a state of the research on the principal techniques used to eliminate details from CAD geometry. The second part introduces the model of idealization proposed. A data-processing implementation, using "Open CASCADE", of the algorithm proposed allows showing, on some examples of mechanical parts, the time savings as well as the percentage of computation results' error before and after CAD geometry's idealization. That contributes enormously to the reduction of design time and the cost price of the product.

\section{State of the art}

The process of a CAD model's preparation passes by a phase of idealization or simplification of the geometry. This phase consists in details elimination whose role, towards simulation, does only increase the time of simulation without any improvements to the quality of simulation results (Benhafida et al., 2005).

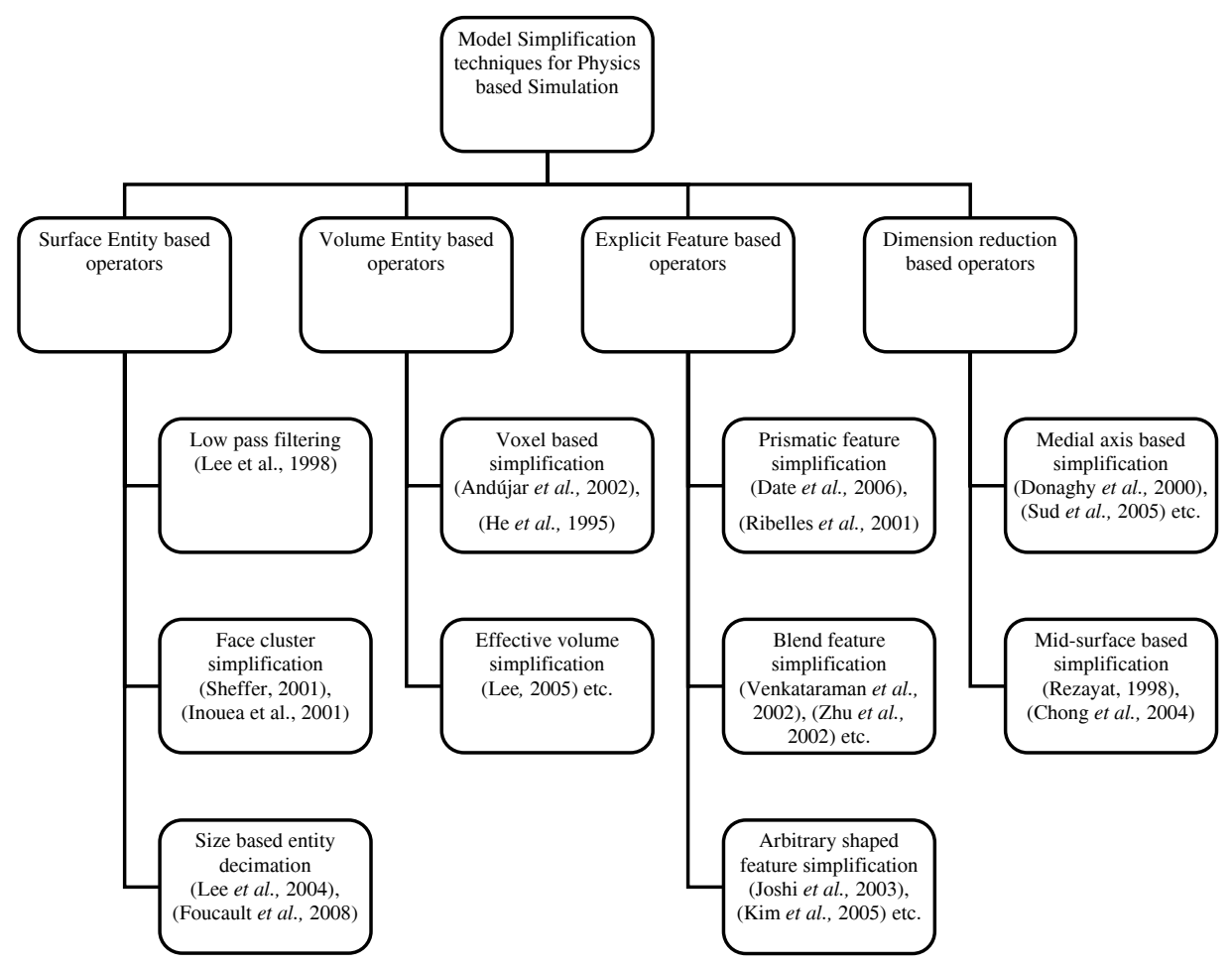

Figure 1. Taxonomy of model simplification techniques (Thakur et al., 2009) 
The review of the literature in the geometry idealization field allows enumerating several techniques and is possible to structure these works according to the Figure 1 suggested by (Thakur et al., 2009). In his works they studied existing model simplification techniques that are useful from physics based simulation and classified them broadly into four main categories based up on the type of simplification operators used in the respective techniques, i.e. surface entity, volumetric entity, explicit feature and dimensional reduction.

It's clearly revealed that there is no single technique can solve all the model simplification problems. For example, the majority of the techniques are applicable for FEA model preparation, whereas the others are suitable for fluid flow problems, collision detection or even as pre-processing steps in recognizing complex features. The factors, namely, application domain, types of features handled, input format, level of automation and type of operators used, need to be taken into consideration before selecting a particular technique. In the following paragraphs we list some principal techniques.

\subsection{Extraction and suppression of the fillets}

Venkataraman (Venkataraman et al., 2002) proposes powerful algorithms to extract the fillets. In his work, the algorithm of suppression predicts final topology by using the structure of the fillets chains, and then finds a geometrical solution based on the intersection of support faces of the fillet. The final topology is deduced by contraction of edges E1 and E2 in vertex (Figure 2a), then by the fusion of the two edges of the face in only one edge and the removal of the face of connection (Figure 2b). (Zhu et al., 2002) and (Venkataraman et al., 2001) are in the same category.

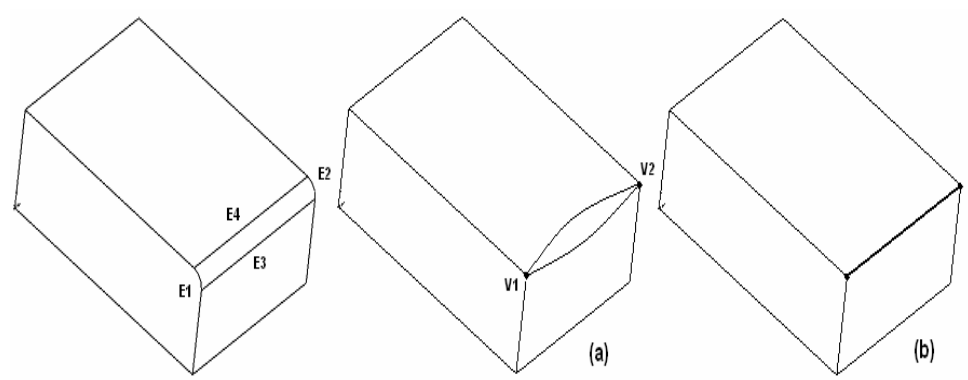

Figure 2. Deduction of final topology by the use of Euler operators 


\subsection{Boolean operators of simplification}

The operators of merging and collapse allowing to remove details of 3D geometries are: merge faces, merge edges, kill void, kill holes, collapse face to edge, collapse face to vertex (Figure 3).

The operators of Euler proposed by Armstrong (Armstrong et al., 1996) are used to remove the shift between two faces and to produce a convex face. That's why two vertexes are created on the edge in order to create a face and to start up an operation of contraction of this face towards the edge.
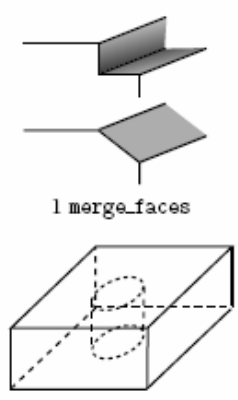

4 kill hole

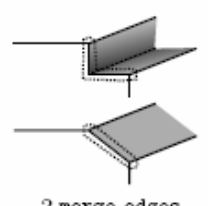

1.

2.

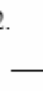

5 collapse_face_to_ed
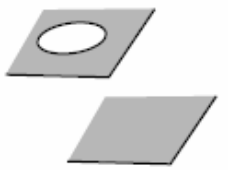

3 kill_void

1.

2.

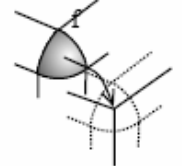

6 collapse_face_to_vertex

Figure 3. Euler Operators for details suppression (Armstrong et al., 1996)

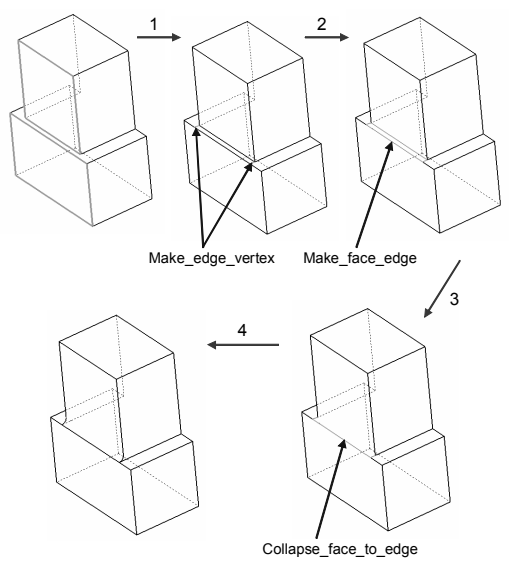

Figure 4. Different suppression stages of faces shift 


\subsection{Virtual topology and regrouping of faces}

Virtual topology consists in regrouping faces in regions (Figure 4). That allows, for example, making mesh "multi-faces" (Sheffer et al., 1997), provided that the faces define an enough plane surface. The criteria of regrouping faces in region are thus mainly the flatness of the region. So this method allows removing the low-size faces by merging them with their adjacent faces or by contracting them in edges. It cannot be considered as a technique of simplification of the geometry but rather as a particular method of mesh.

\subsection{Mesh Stress Topology}

(Foucault et al., 2008), reported a topology simplification technique for finite element mesh generation. The authors have developed a Mesh Stress Topology (MCT) based on model simplification scheme to address the sharp corner matching requirements. MCT entities are defined as composite topological entities created to suit mesh generation requirements stated before. The MC face is a poly-surface, defined as the union of Riemannian surfaces constituting the reference model. The $\mathrm{MC}$ edge is a poly-curve, defined as a union of riemannian curves constituting the reference model. The MC entities are used to represent the model because they preserve the exact geometries as they are a higher level representation of the low level B-Rep entities that define the reference model.

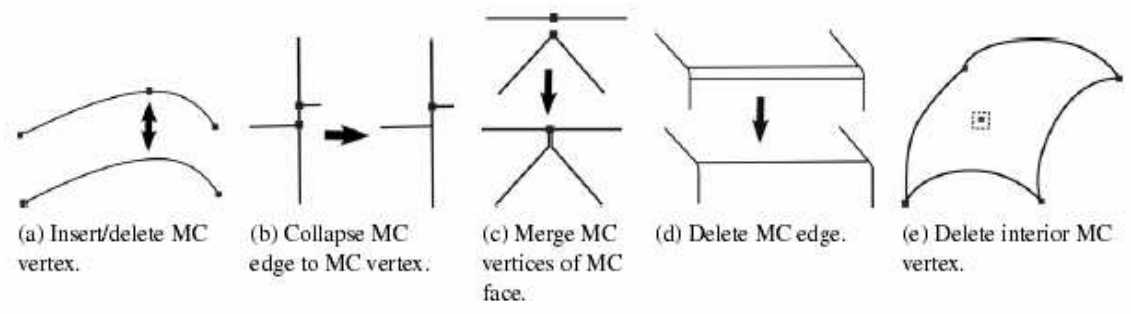

Figure 5. MCT operators (Foucault et al., 2008)

The authors have implemented graph based operators for deleting $\mathrm{MC}$ edge and vertex, inserting $\mathrm{MC}$ vertex in $\mathrm{MC}$ edge, collapsing $\mathrm{MC}$ edge to $\mathrm{MC}$ vertex and merging MC vertices for simplification. The mesh quality stress for MC entities is decided by its size and curvature. The size for an $\mathrm{MC}$ face is defined as distance between face boundaries while for an MC edge as length of the edge. Redundant edges situated in planar regions need to be deleted using $\mathrm{MC}$ delete operator. $\mathrm{MC}$ vertex deletion and MC edge collapse operators are used to get rid of small MC edges. Constricted sections in MC faces are suppressed by using MC vertex insertion and vertex merging operators (Figure 5). The mesh quality constraints used by the authors are mesh element size and discretization error. The mesh element size is represented by 
using over density ratio which is the ratio of the size map to the effective element size. The discretization error is a measure of the gap between the mesh and the geometry. Consequently this technique is considered as a specific method of meshing.

\subsection{Using of form features}

In this framework, the features are functions of geometric model's creation. They aim at coupling, geometrical and semantic information (hole, groove, fillet, etc.) in only one entity. These entities, having strong geometric components, support suitable significances for a trade or a specific application (design, manufacture, analysis, etc.). The features can be used in modelling or extraction. For example, the entity hole can be interpreted within the meaning of analysis like carrier of boundary conditions or concentrator of stress, etc. The suppression of a geometric form consists simply on eliminating it from the creation tree of the part (Belaziz et al., 2000) and (Venkataraman et al., 2002). So each actor intervening in the product's life cycle has his own language of description and interpretation based on form features. The extraction of features of forms in a process of simplification of the geometry passes by two steps. At first, it consists on identifying the associated characteristic and topological parameters (faces, edges and vertexes). In the second time, it is a question of reconstituting the geometrical model without the removed forms (Figure 6) (Joshi et al., 1988) and (Venkataraman et al., 2001).

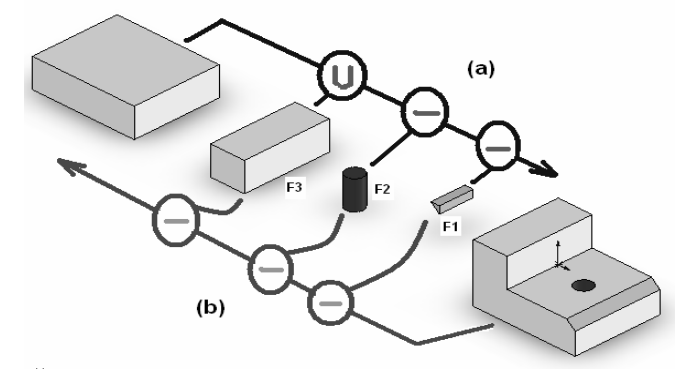

Figure 6. (a) Addition of forms features (b) Extraction of forms features

\subsection{Medial axis and surfaces transformation}

The "Medial Axis Transformation" was proposed by Armstrong (Armstrong et al., 1996), Bridgett and Donaghy (Donaghy et al., 2000) to carry out the simplification and the idealization of B-Rep geometries. The MAT makes it possible to build a skeleton of B-Rep model. In the case 2D geometry (Figure 7), to obtain the median axis, a circle of variable diameter sweeps the interior of the geometry in 
order to keep a contact with contour at least 2 points. The skeleton is obtained by building the places of the centres.

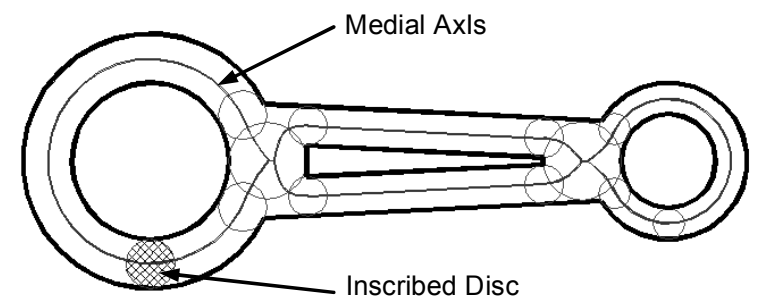

Figure 7. Medial axis

For a 3D geometry, a sphere with a variable diameter sweeps the interior of volume. The place of the center of this sphere represents a surface (Figure 8). This skeleton is then used to carry out an analysis of the geometry in order to characterize the whole of details which make it up (Donaghy et al., 2000), (Rezayat, 1998) and (Armstrong et al., 1996).

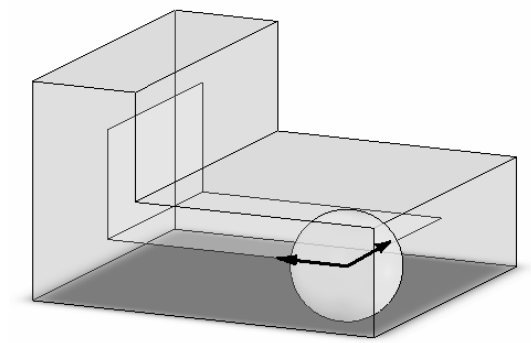

Figure 8. Median Surface

\section{Discussion}

Based on the bibliography presented in Section 2, our contribution is positioned in the first colon of the Figure 1 proposed by (Thakur et al., 2009): "model simplification technique for surface entity based operators". Our method is distinguished by:

- In our idealization process the input and output files are in a STEP format, which is a neutral format (used by the totality of CAD systems).

- Our idealization process is interactive. After treatment, the CAD part is presented by an iso-zone model, so the designer can intervene in the choice of details to be deleted using some criteria. 
- Our idealization process is based on a CAD model, the reconstruction process is performed without approximation.

- A hierarchic link is saved between the initial model and the adapted model.

- The idealized model can be used in a multiple case study: Finite element analysis, Assembly process, Detection of collisions, Dynamic analysis, etc. because of the output format type (STEP format).

\section{Algorithm of idealization}

The proposed algorithm composed of three principal interdependent stages represented in Figure 9. Each stage implies overlapping algorithms.

\subsection{Principle}

In order to be independent of the $\mathrm{CAD} / \mathrm{CAM}$ systems, the proposed algorithm relies on a neutral file (Standard for Exchange of Product (STEP) to import the data of the part to be idealized. To use various tools of simulation, the idealized CAD geometry will be also stored under the same neutral format (STEP). The stage (1) of the algorithm consists on a phase of pre-treatment of CAD model. It allows restructuring the Boundary Representation (B-rep) model of the part to a data base. The structured information in this data base relates to the faces, the wires, the edges and the vertex which includes the geometry model of the part. The stage (2) consists in identifying the details candidates for elimination. That implies the implementation of algorithms of identification based on criteria (forms, sizes...). The result of this phase is a representation of Iso-zones (comparable with the representation of the Isovalues of stress, deformations in the computational tools by finite elements method) targets for elimination. These Iso-zones are entities (edges, faces) coloured according to a gradient of criticality. This original vision enables the designer to visualize the least influential zones (high order of criticality) on the computation results, giving him the possibility either to inter-actively eliminate the entities which have high order of criticality, or to apply the automatic algorithms of elimination. The stage (3) consists in removing the identified details, then in rebuilding the geometric model after suppression. The result of this phase is an idealized CAD model whose elementary topology is valid. At the exit of the algorithm, the designer has at his disposal an idealized model recorded in format STEP for a simulation by finite elements. The algorithms associated at the above mentioned stages are developed in the following sections. 


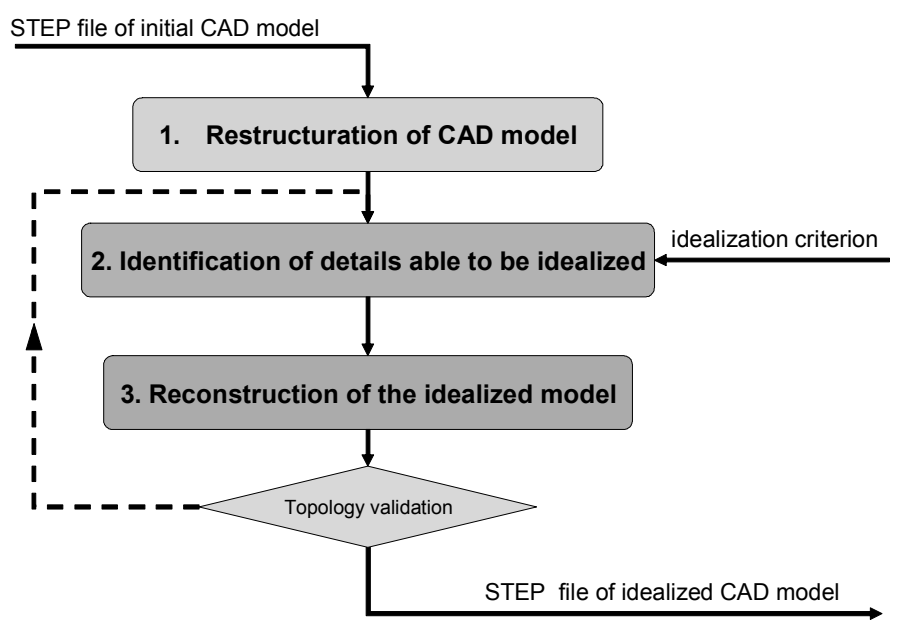

Figure 9. Algorithm of idealization

\subsection{Stage 1: Restructuration of CAD model}

The restructuration of the CAD model aims at exploring and making it easy to handle the topological and geometrical data associated with part. Figure 10 presents the algorithm associated with this stage.

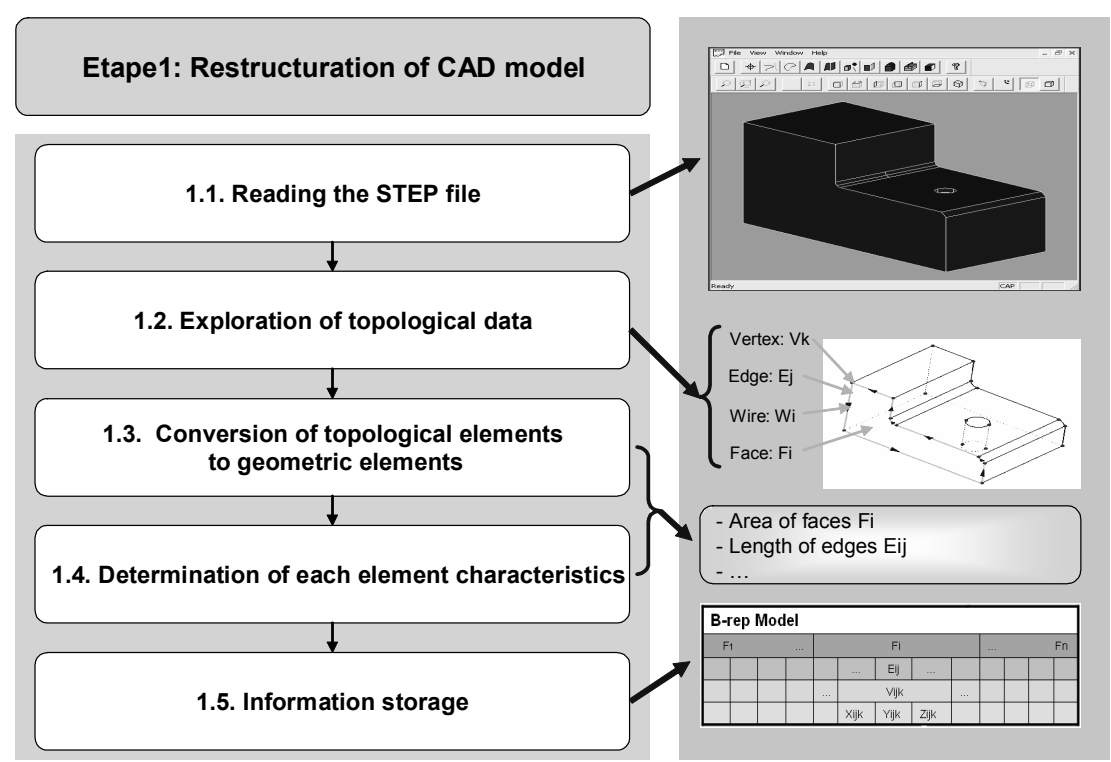

Figure 10. Restructuration algorithm of CAD model 
Reading the STEP file led to determine the characteristics of each element of Brep model, namely

- vertex: (cordinates),

- Edge: (associated vertex, length),

- wire: (associated edges, orientation, length),

- Face: (associated wires, normal, area).

This information will be stored in a data base allotted to the part. Any geometrical handling on the part implies the processing of information indexed in the data base. For a given face, it is evident to know the associated wires, the participating edges also the list of vertex appearing in the face. Geometrical information such as perimeter and the surface of the face are directly deduced.

\subsection{Stage 2: Identification of details}

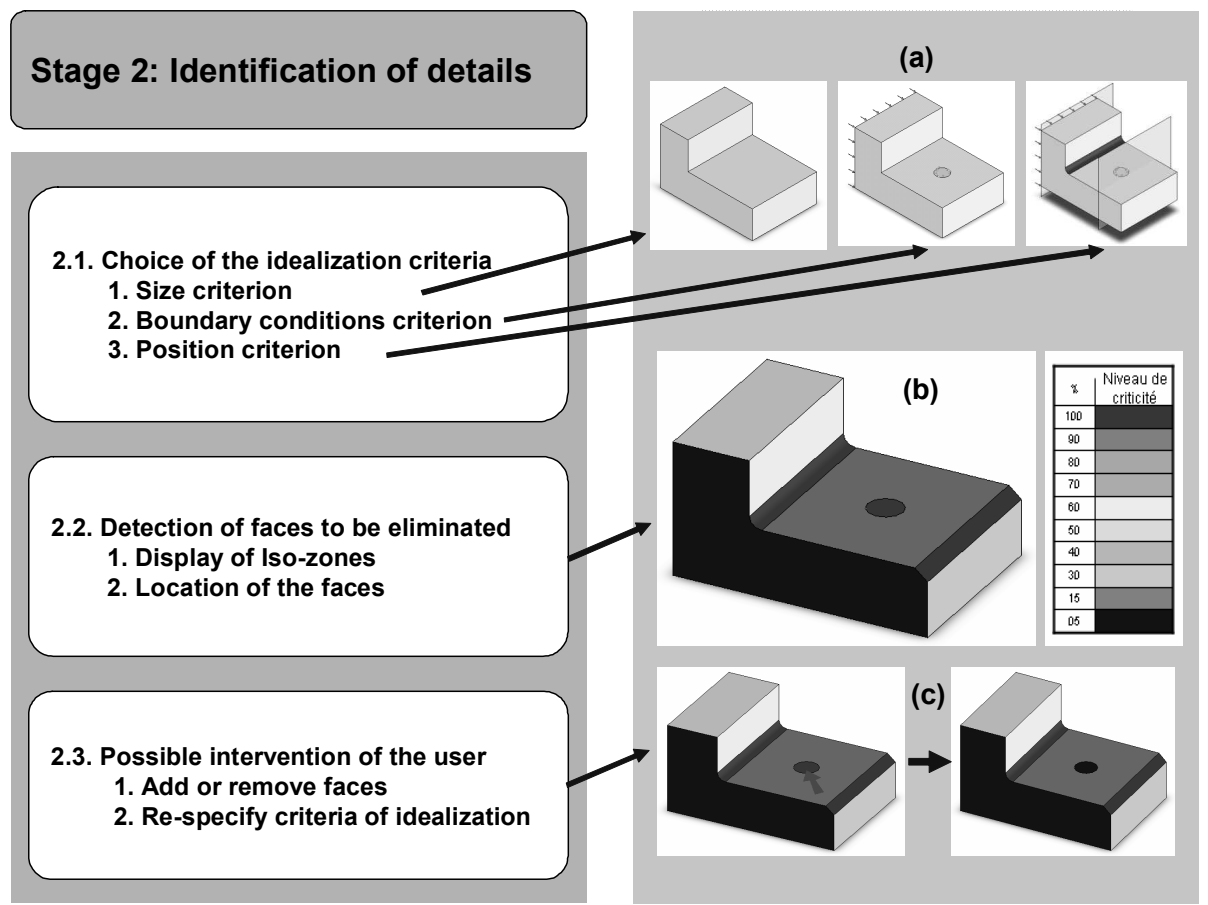

Figure 11. Detail identification algorithm 
On the basis of the collected information in the preceding stage, the designer has to specify an idealization criterion (Figure 11a). Three criteria are already identified. The criterion of size relates to the lengths of the edges and the areas of the faces. An increasing or a decreasing sort, on the faces and the edges, is quickly carried out starting from the data base of part. The procedure of sort consists in studying the ratio of edge's lengths to the longest edge and the ratio of faces' surfaces to the largest face. The Boundary Conditions (BC) criterion does not allow removing the details which have BC. Currently, details that have BC are specified by the designer. These details will be automatically eliminated from the list of the details candidates to elimination. The criterion of the detail's position compared with the loading allows keeping details, if they are regarded as concentrators of stress because of their orientations compared with the loading, even if they are eligible from the size point of view. This criterion is not taken into account to date.

Once criteria are specified, the detection's result of the eliminating details is represented by Iso-zones (Figure 11b). Faces and Edges represented by sharp colors are target to elimination (high level of criticality). The designer has finally the interactive choice to eliminate or keep the details. He can also add other faces (or edges) to the list of the entities to eliminate (Figure 11c). This stage is easy because, the designer has an access to the B-rep model of the part.

\subsection{Stage 3: Suppression of details and rebuilding of idealized CAD model}

The objectives of this stage are to remove the details identified in the previous section then rebuild the idealized part (Figure 12). The procedure of CAD model's rebuilding, after the elimination of faces and edges, consists, firstly, in detecting the adjacent faces to the removed entities. The detection of adjacent faces is based on the database part processed in the first stage (Figure 12b). Secondly, it consists in prolonging these faces in all directions (Figure 12c). That will lead to detect intersections curves due to the prolongation of faces (Figure 12d) Vertex 'limit' will be determined from curves (Figure 12e), then the edges will be delimited by vertex (Figure 12f). CAD model is regenerated by rebuilding faces associated to new wires (Figures $12 \mathrm{~g}$ and $12 \mathrm{~h}$ ). A new database of the idealized part is thus completed. The designer has the B-rep model of the part before and after idealization. A file 'STEP' is finally generated in order to use the idealized CAD model by an analysis tool.

The proposed algorithm of idealization is adjudicated universal because it is based only on the B-rep model of the CAD geometry. This algorithm is completely independent of the CAD geometry creation tree, because of the non-uniqueness of this tree. In addition, CAD model, once exported, loses any information in its manner of creation. Attempts at the re-creation of Constructive Solid Geometry (CSG) model exist, in fact the module Feature Works of SolidWorks, but the results obtained are not very conclusive. 


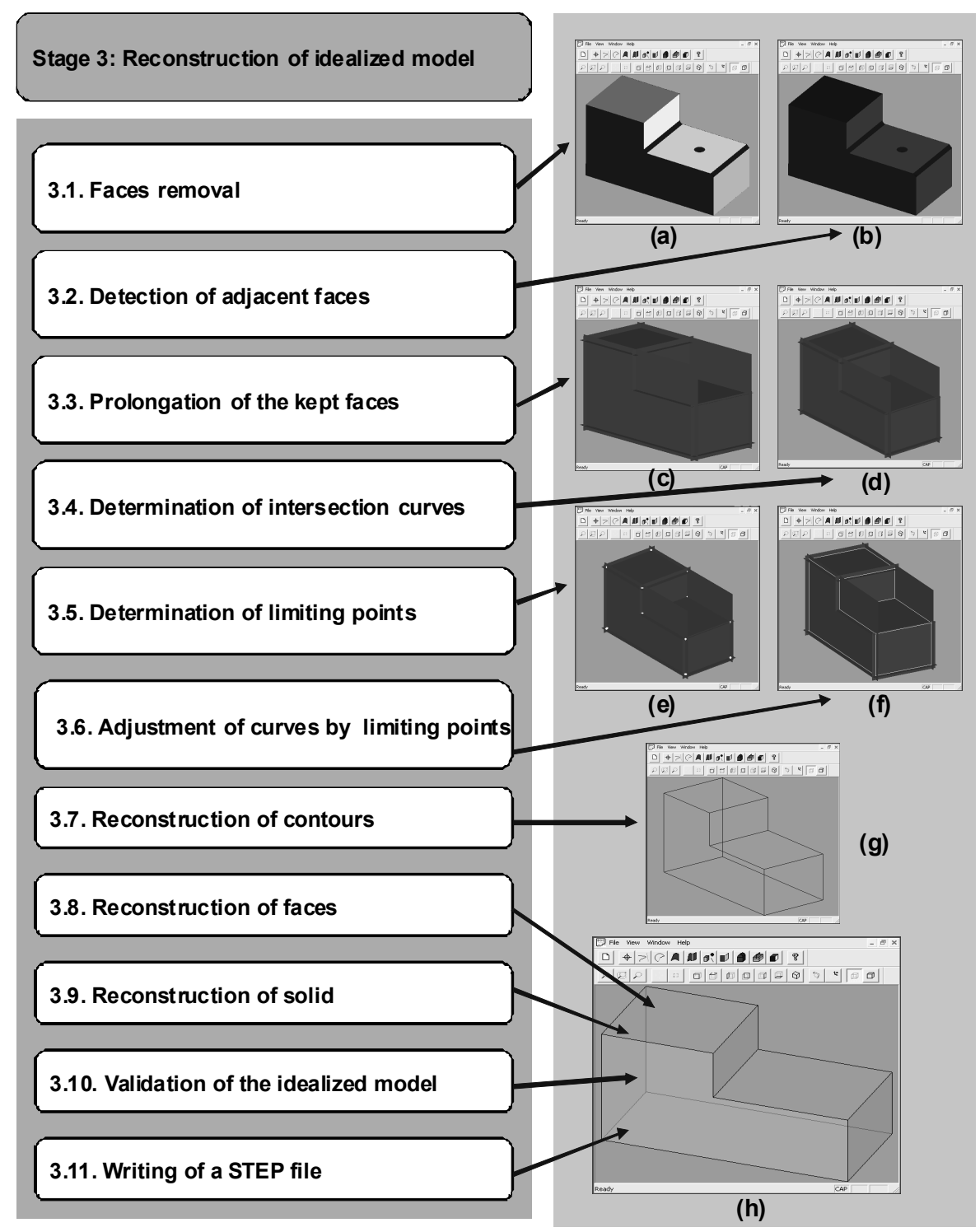

Figure 12. Algorithm of details suppression \& rebuilding of idealized CAD model 


\section{Implementation and validation}

\subsection{Implementation}

The data-processing implementation of the idealization algorithm was carried out on development's platform, "Open Cascade". Open Cascade is an environment dedicated to the development of 3D applications of CAD-CAM multi-platforms. This platform is available and free on Internet. It is based on a bookstore of $\mathrm{C}++$ classes and tools developed and available in open source.

\subsection{Validation examples}

In this section, two examples of validation will allow validating the principal functionalities of the idealization algorithm. Figure 13 presents two supports. These parts are selected because they have a broad variety of mechanical parts in terms of its forms, the boundary conditions, and also the details which they contain.

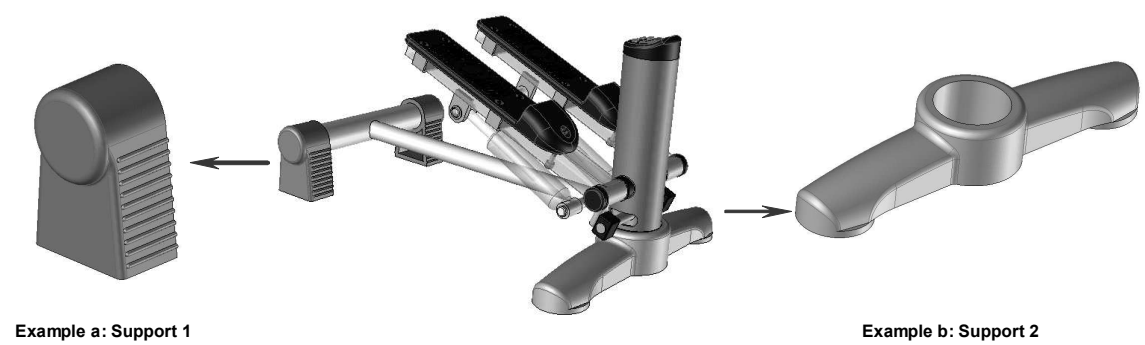

Figure 13. Validation example

Figure 14 presents the illustration of the principal stages to pass from a CAD model of the support 1 (Figure 14a), where boundary conditions are defined (loading and fixing), to a model of analysis whose geometry is idealized (Figure 14e). A very important stage (Figure 14c) represents the support 1 by Iso-zones. These izo-zones give to the designer a very clear idea of the details candidates to elimination by sharp colors, according to the level of criticality (going from $0 \%$ to $100 \%$ ).

Figure 15 also represents the support 2 by Iso-zones. It shows also the principal step leading to the idealized model of the support.

Figure 16 represents two Open Cascade windows in which, are represented, the idealized CAD models of the support 1 as well as the support 2. These models will be saved in 'STEP' format to be simulated by the finite element method. 


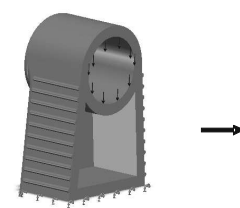

(a)

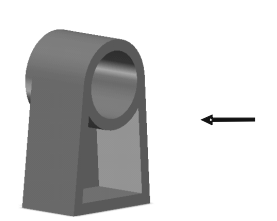

(e)

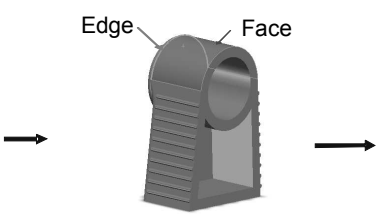

(b)

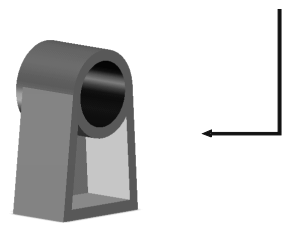

(d)

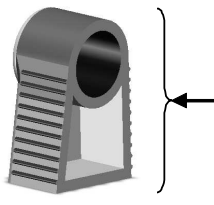

(c)

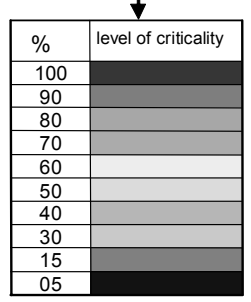

Figure 14. Representation by Iso-zones of the support 1

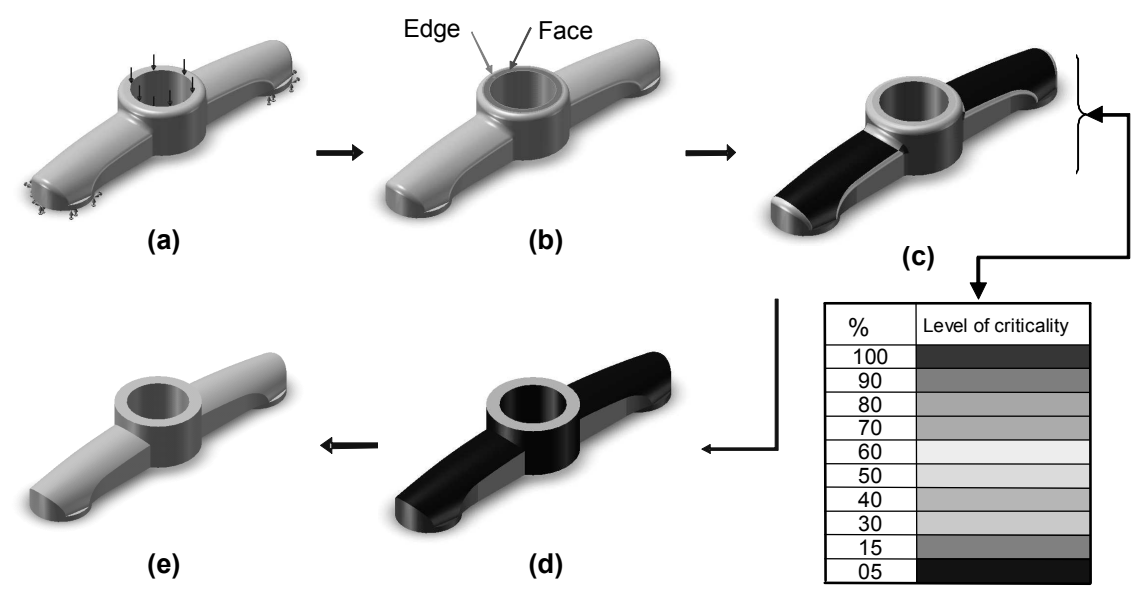

Figure 15. Representation by Iso-zones of the support 2 


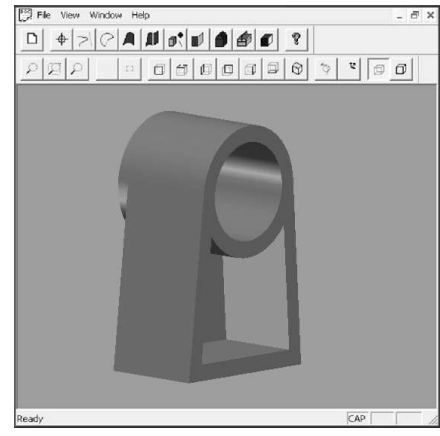

(a) : Idealized model of the support 1

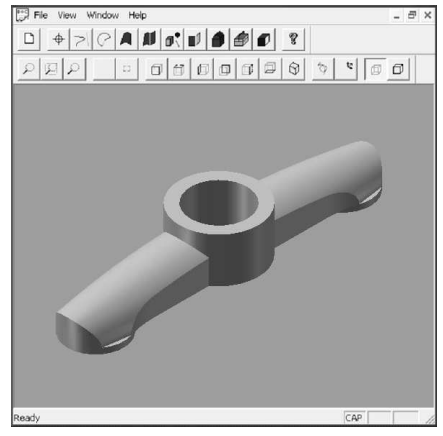

b : Idealized model of the support 2

Figure 16. Result of the idealization algorithm implemented by Open Cascade

Table 1 represents the number of the B-rep entities of the support 1 and the support 2, before and after idealization. It's shown that the number of entities after idealization is lower than that before idealization. That generates a reduction of the mesh and computation times of the idealized model.

Table 1. The B-rep entities before and after idealization

\begin{tabular}{|c|c|c|c|c|c|c|c|c|}
\cline { 2 - 9 } \multicolumn{1}{c|}{} & \multicolumn{2}{c|}{ Number of Faces } & \multicolumn{2}{c|}{ Number of Wires } & \multicolumn{2}{c|}{ Number of Edges } & \multicolumn{2}{c|}{ Number of Vertexes } \\
\cline { 2 - 9 } \multicolumn{1}{c|}{} & Initial Model & Idealized Model & Initial Model & Idealized Model & Initial Model & Idealized Model & Initial Model & Idealized Model \\
\hline Support 1 & 77 & 14 & 81 & 18 & 194 & 27 & 120 & 16 \\
\hline Support 2 & 207 & 142 & 222 & 156 & 572 & 399 & 368 & 260 \\
\hline
\end{tabular}

Figures 17 and 18 represent the computation results by finite elements method of the support 1 and the support 2 before and after the elimination of details. In order to perform the FE analysis, we use the below conditions:

\section{- Support 1:}

- Analysis type: linear elastic behaviour;

- Material: PVC;

- Boundary conditions: \{ F11: Imposed displacement; F12: Force: F=500N \}.

\section{- Support 2:}

- Analysis type: linear elastic behaviour;

- Material: PVC; 
- Boundary conditions: \{ F21 \& F22: Imposed displacement ; F23: Force: $\mathrm{F}=1000 \mathrm{~N}\}$.

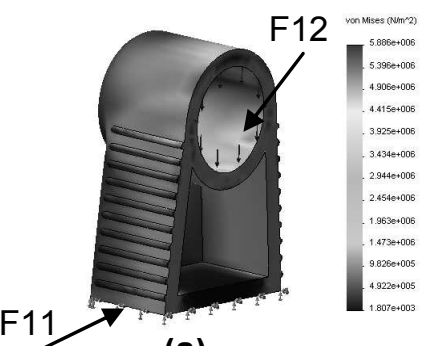

(a)

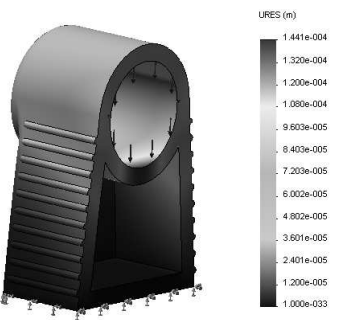

(b)

\begin{tabular}{|l|c|c|c|}
\hline & Initial Model & Model idealized & difference \\
\hline Van Mises Stress Max (MPa) & 5.886 & 5.444 & $7,5 \%$ \\
\hline Displacement Max (mm) & $1.44110^{-1}$ & $1.45510^{-1}$ & $0,9 \%$ \\
\hline Simulation Time (s) & 28 & 9 & $68 \%$ \\
\hline
\end{tabular}

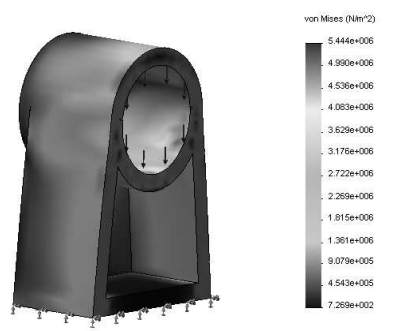

(c)

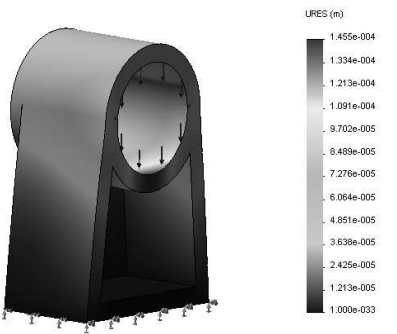

(d)

Figure 17. Computation results of the support 1

Figures $17 \mathrm{a}$ and $\mathrm{b}$ represent respectively the states of stress and the displacements of the support 1 before the application of the idealization algorithm. The Figures $17 \mathrm{c}$ and d respectively show the states of stress and displacements parts after idealization. We notice that the time saving of computation is $68 \%$. The error relating to the values of displacements is $0.9 \%$, while that of the equivalent stress is $7.5 \%$. For the support 2 , we represent respectively on Figures $18 \mathrm{a}$ and $18 \mathrm{~b}$ the states of stress and displacements before the application of the idealization algorithm, whereas they are represented respectively on Figures $18 \mathrm{c}$ and $18 \mathrm{~d}$ but this time after the idealization of parts. It is noted that the time saving of computation is $62.5 \%$ and the error related to the values of displacements is $2.13 \%$, while that of the equivalent stress is $1.36 \%$. For a preliminary dimensioning analysis, this error is considered to be acceptable. If the designer aims at having a much more precise analysis in order to check the chosen dimensions, he can apply more strict criteria of idealization to the dimensions of the 
details to be removed or to the site of the details compared to the loading. This won't allow removing the forms which are the concentrator of stress. In all cases, the taking into account of the idealization link in "design-analysis" chain brings an important saving of computing time without any significant loss in the quality of the results. We must notice that this procedure of idealization requires a negligible execution time compared to the total simulation time.

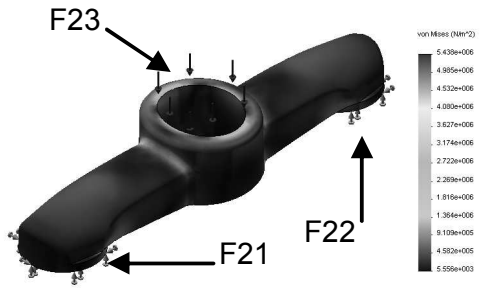

(a)

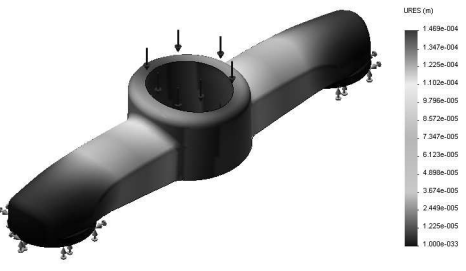

(b)

\begin{tabular}{|l|c|c|c|}
\hline & Initial Model & Model idealized & difference \\
\hline Van Mises Stress Max (MPa) & 5.438 & 5.364 & $1,36 \%$ \\
\hline Displacement Max (mm) & $1.46910^{-1}$ & $1.50110^{-1}$ & $2,13 \%$ \\
\hline Simulation Time (s) & 32 & 12 & $62,5 \%$ \\
\hline
\end{tabular}

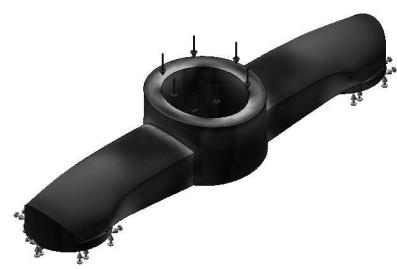

(c)

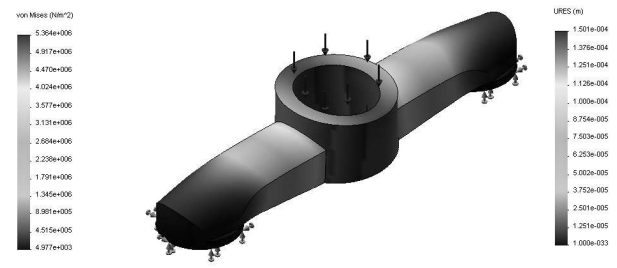

(d)

Figure 18. Computation results of the support 2

\section{Conclusion}

This paper presents an idealization algorithm of CAD models for a simulation by the finite element method. The algorithm proposed consists on reading the B-rep model of the CAD geometry in order to identify, then remove the details considered to be superfluous for mechanical analysis. In this work, the creation tree of CAD model (CSG) is not taken into account not only because it is not unique but also it is easily lost by a simple CAD model transfer between different working tools.

The examples of validation show that a suitable elimination of the details in a CAD model allows saving a very important time (up to $70 \%$ ) in the procedure of 
simulation while keeping a high quality of the computation results (an error which do not exceed 2\%).These observations were found by doing simulations by finite elements before and after idealization. This work obeys perfectly with the current tendencies of the industrialists who are interested more and more in preparing CAD models during the pre-processing phase.

In the future, it is important to consider other criteria of idealization such as the position of the detail compared to the boundary conditions (BC). Now, the designer is the one who has to check the list of details to be removed in order to keep those which are carrying $\mathrm{BC}$, also those which are placed in the direction of the loading. Information on $\mathrm{BC}$, are now, specified since the design phase. However, they do not yet appear in the STEP export file.

\section{References}

Aifaoui N., Intégration $\mathrm{CAO} / \mathrm{Calcul}$ : une approche par les features de calcul, Thèse de Doctorat, Université de Valenciennes, 2003.

Andújar C., Brunet P., Ayala D., "Topology reducing surface simplification using discrete solid representation", ACM Transactions on Graphics, vol. 21, n² 2, 2002, p. 88-105.

Armstrong C. G., Donaghy R. J., Bridgett S. J., "Derivation of appropriate Idealisations in Finite Element Modelling", $3^{\text {th }}$ International Conference on Computational Structures technology, Budapest, 1996.

Belaziz M., Bouras A., Brun, "Morphological analysis for product design”, CAD Computer Aided Design, vol. 32, n 5-6, 2000.

Benamara A., Contribution à l'intégration de la composante calcul dans une démarche de conception fonctionnelle intégrée, application aux mécanismes, Thèse de doctorat, université de Valenciennes, décembre 1998.

Benhafida Y., Troussier N., Boudaoud N., and Cherfi Z., « Méthode d'aide à l'idéalisation de modèles issus de la CAO pour le calcul de structures ", Mecanique \& Industries, vol. 6, 2005.

Chong C. S., Kumar A. S., Lee K. H., "Automatic solid decomposition and reduction for nonmanifold geometric model generation”, CAD Computer Aided Design, vol. 36, n 13, 2004, p. 1357-1369.

Date H., Kanai S., Kishinami T., Nishigaki I., "Flexible feature and resolution control of triangular meshes", Proceedings of the Sixth IASTED International Conference on Visualization, Imaging and Image Processing, Palma de Mallorc, Spain, 2006.

Donaghy R. J., Armstrong C. G., Price M. A., "Dimensional reduction of surface models for analysis", Engineering with computers, vol. 16, n 1, 2000, p. 24-35.

Foucault G., Cuillière J., François V., Léon J., “Adaptation of CAD model topology for finite element analysis", CAD Computer Aided Design, vol. 40, n² 2, 2008, p. 176-196. 
Hamdi M., Aifaoui N., Benamara A., «Etat de l'art des méthodes d'idéalisation en CAO », CMSM'07, Monastir 2007.

He T., Hong L., Kaufman A., Varshney A., Wang S., "Voxel based object simplification", Proceedings of Visualization'95, Atlanta. GA, 1995.

Inouea K., Itoha T., Yamadaa A., Furuhatab T., Shimadac K., "Face clustering of a largescale CAD model for surface mesh generation", CAD Computer Aided Design, vol. 33, $\mathrm{n}^{\circ} 3,2001$, p. 251-261.

Joshi S., Chang T. C., "Graph-based heuristics for recognition of machined features from a 3d solid model”, CAD Computer Aided Design, vol. 20, n² 2, 1988, p. 58-66.

Joshi N., Dutta D., "Feature simplification techniques for freeform surface models", Journal of Computing and Information Science in Engineering, 3, 2003, p. 177-186.

Kim S., Lee K., Hong T., Kim M., Jung M., Song Y., "An integrated approach to realize multi-resolution of B-Rep model", Proceedings of the 2005 ACM Symposium on Solid and Physical Modeling, Cambridge, MA, 2005.

Lee J. Y., Lee J. H., Kim H., Kim H. S., "A Cellular topology-based approach to generating progressive solid models from feature-centric models", CAD Computer-Aided Design, vol. $36, n^{\circ} 3,2004$, p. $217-229$.

Lee S. H., "A CAD-CAE integration approach using feature-based multiresolution and multiabstraction modeling techniques", CAD Computer Aided Design, vol. 37, n 9, 2005, p. $941-955$.

Lee S. H., "Feature-based multiresolution modeling of solids", ACM Transactions on Graphics, vol. 24, n 4, 2005, p. 1417-1441.

Lee Y. G., Lee K., "Geometric detail suppression by the Fourier transform”, CAD Computer Aided Design, vol. 30, n 9, 1998, p. 677-693.

Louhichi B., Benamara A., François V., "Automatic reconstruction of CAD model from a deformaed mesh", European Journal of Computational Mechanics, vol 18, n 3-4, 2009, p. $177-194$.

Rezayat M., "Midsurface abstraction from 3d solid models : general theory and applications", CAD Computer Aided Design, vol. 28, n 11, 1998.

Ribelles K., Heckbert P. S., Garland M., Stahovich T., Shivastava V., "Finding and removing features from polyhedra", Proceedings of ASME DETC'01, Pittsburgh, PA, 2001.

Sheffer A., "Model simplification for meshing using face clustering", CAD Computer Aided Design, vol. 33, n 13, 2001, p. 925-934.

Sheffer A., Blacker T. D., Clustering M., "Automated detail suppression using virtual topolgy", ASME, 1997, p. 57-64.

Sud A., Foskey M., Manocha D., "Homotopy-preserving medial axis simplification", Proceedings of the 2005 ACM symposium on Solid and physical modeling, Cambridge, MA, 2005. 
Thakur A., Banerjee A.G., Gupta S.K., "A survey of CAD model simplification techniques for physics-based simulation applications", CAD Computer Aided Design, 41, 2009, p. $65-80$.

Venkataraman S., Sohoni M., "Reconstruction of feature volumes and feature suppression", Proceedings of the seventh acm symposium on solid modeling and applications, ACM Press, 2002, p. 60-71.

Venkataraman S., Sohoni M., Elber G., "Blend recognition algorithm and applications", Proceedings of the sixth acm symposium on solid modeling and applications, ACM Press, 2001, p. 99-108.

Venkataraman S., Sohoni M., Kulkarni V., "A graph-based framework for feature recognition", Proceedings of the sixth acm symposium on solid modeling and applications, ACM Press, 2001, p. 194-205.

Venkataraman S., Sohoni M., Rajadhyaksha R., "Removal of blends from boundary representation models", Proceedings of the seventh acm symposium on solid modeling and applications, ACM Press, 2002, p. 83-94.

Westphal M., L'avenir de l'analyse mécanique, CAD Magazine, vol. 126, 2005, p. 23-25.

Zhu H., Menq C. H., "B-rep model simplification by automatic fillet/round suppressing for efficient automatic feature recognition", CAD Computer Aided Design, vol. 34, n 2, 2002, p. 109-123.

Received: 18 May 2009 Accepted: 15 February 2010 
